\title{
Impact of an Intravenous Trimethoprim/Sulfamethoxazole Shortage on Treatment Outcomes Among HIV-Infected Patients with Pneumocystis jirovecii Pneumonia
}

\author{
Thomas J. Dilworth, PharmD, BCPS, AAHIVP; Omar M. Ibrahim, PhD; \\ and Renée-Claude Mercier, PharmD, BCPS, PhC
}

\begin{abstract}
BACKGROUND: Trimethoprim/sulfamethoxazole (TMP/SMX) is the recommended first-line treatment for human immunodeficiency virus (HIV)infected patients with Pneumocystis jirovecii pneumonia (PJP). However, in June 2010, the lone manufacturer of intravenous (IV) TMP/SMX in the United States stopped production of this medication.
\end{abstract}

OBJECTIVES: To (a) evaluate the impact of the national IV TMP/SMX shortage on PJP treatment outcomes between 2 groups of HIV-infected patients-those treated before the shortage and those after the shortage-and (b) compare the length of hospital stay (LOS) and PJP treatment used before and after the shortage.

METHODS: A retrospective, quasi-experimental study examining 2 groups of HIV-infected adult patients with PJP was performed at an academic medical center from September 1, 2008, to June 30, 2012. Patients treated when IV TMP/SMX was available, or preshortage (PRE), were compared with patients treated when IV TMP/SMX was not available, or postshortage (POST). PRE included patients treated between September 1, 2008, and May 30, 2010, and POST included patients treated between June 1, 2010, and June 30, 2012.

RESULTS: Thirty-six patients were included in the study, 18 in each group. Treatment failure, the primary outcome, included mortality or worsening clinical status (WCS) after at least 5 days of therapy. Three patients in PRE (16.7\%) and 6 patients in POST (33.3\%) experienced treatment failure $(P=0.248)$. No patients in PRE and 3 patients in POST (16.7\%) experienced WCS $(P=0.035)$. Three patients in each group expired. In POST, 5 of the 6 treatment failures $(83.3 \%)$ occurred during the first 6 months of the shortage. Median (interquartile range) LOS was 11 days (7-17) in PRE and 14 days (5-22) in POST $(P=0.800)$. In PRE, 7 patients $(38.9 \%)$ were initiated on oral PJP treatment compared with $13(72.2 \%)$ in POST $(P=0.042)$.

CONCLUSIONS: The national shortage of IV TMP/SMX may have led to an immediate but temporary negative impact on treatment outcomes among HIV-infected patients with PJP at an academic medical center. Pharmacist collaboration with physicians may have helped mitigate the impact of this drug shortage on patient outcomes.

J Manag Care Pharm. 2014;20(12):1246-54

Copyright $\odot 2014$, Academy of Managed Care Pharmacy. All rights reserved.

\section{What is already known about this subject}

Anti-infective drug shortages are common and may compromise patient care.

Intravenous trimethoprim/sulfamethoxazole (IV TMP/SMX) is the treatment of choice for human immunodeficiency virus (HIV)infected patients with moderate-to-severe Pneumocystis jirovecii pneumonia (PJP).

In 2011, a survey of infectious diseases physicians found that IV TMP/SMX was the most common anti-infective on shortage and that this shortage led to the use of less effective medications such as pentamidine for the treatment of PJP.

\section{What this study adds}

This is the first study describing the impact of the IV TMP/SMX shortage on treatment outcomes among HIV-infected patients with PJP.

The IV TMP/SMX shortage had an immediate but temporary negative impact on treatment outcomes among HIV-infected patients with PJP.

Pharmacist collaboration with physicians may have helped mitigate the negative impact of the IV TMP/SMX shortage on treatment outcomes among HIV-infected patients with PJP.

$\mathrm{P}$ neumocystis jirovecii pneumonia (PJP) is an opportunistic infection caused by the environmental fungi Pneumocystis jirovecii. ${ }^{1,2}$ Before the advent of highly active antiretroviral therapy (HAART) and the use of PJP chemoprophylaxis, PJP used to be a leading cause of morbidity and mortality among human immunodeficiency virus (HIV)-infected patients with acquired immune deficiency syndrome (AIDS); however, the number of HIV-infected patients with PJP has declined in recent years. ${ }^{3,4}$ Even with HAART and PJP chemoprophylaxis, PJP is still the second most common opportunistic infection among HIV-infected patients in the United States and is responsible for 3.9 infections per 1,000 person years. ${ }^{4}$ High-dose trimethoprim-sulfamethoxazole (TMP/SMX) is firstline therapy for HIV-infected patients with PJP and intravenous (IV) TMP/SMX is recommended for patients with moderate-tosevere PJP. ${ }^{5}$ 
In June 2010, the lone manufacturer of IV TMP/SMX in the United States halted its production of this medication due to manufacturing issues. ${ }^{6}$ This led to a national shortage of IV TMP/SMX. ${ }^{6}$ At the University of New Mexico Hospital (UNMH), we were unable to obtain IV TMP/SMX from June 1,2010 , until production of this medication resumed in June 2013. Given that TMP/SMX is the first-line agent for treatment of PJP in HIV-infected patients, we decided to study the impact of this drug shortage on treatment outcomes among HIV patients diagnosed with PJP at our hospital.

Our primary objective was to evaluate the impact of the national shortage of IV TMP/SMX on PJP treatment outcomes among HIV-infected adult patients using a retrospective, quasiexperimental study design, with the hypothesis that HIVinfected patients with PJP experienced worse treatment outcomes after the IV TMP/SMX shortage than before the shortage. Secondary objectives of interest included a comparison of the length of hospital stay (LOS) and PJP treatment used before and after the shortage.

\section{Methods}

\section{Study Participants}

A retrospective chart review of all patients with an International Classification of Diseases, Ninth Revision, Clinical Modification (ICD-9-CM), 136.3, at UNMH between June 1, 2008, and June 30,2012 , was performed. UNMH is a 646-bed, tertiary care hospital located in Albuquerque, New Mexico. Eligible patients were (a) HIV-infected at time of PJP diagnosis, (b) $\geq$ aged 18 years, and (c) diagnosed with moderate-to-severe PJP as confirmed by a Gomori Methenamine Silver or Calcofluor White stain positive for PJP and/or clinician judgment with a room air partial pressure of oxygen $(\mathrm{PaO} 2)<70$ millimeters of mercury $(\mathrm{mmHg})$ or a similar marker of hypoxia. Exclusion criteria included patients who had mild PJP, were admitted on PJP treatment from another facility, were nonadherent to outpatient PJP treatment after hospital discharge, had an allergy to TMP/ SMX, and/or who died $\leq 24$ hours after admission. Patients treated when IV TMP/SMX was available, or preshortage (PRE), were compared with patients treated when IV TMP/SMX was not available, or postshortage (POST). The PRE group included patients treated between September 1, 2008, and May 30, 2010 , and the POST group included patients treated between June 1, 2010, and June 30, 2012. This study was approved by the UNM Health Sciences Center Human Research Review Committee and conforms to the STROBE (Strengthening the Reporting of Observational Studies in Epidemiology) recommendations for reporting observational studies. ${ }^{7}$

\section{Data Collection}

Data from participants meeting the inclusion criteria were retrospectively collected from the electronic medical record using a systematic data collection form. The following data were collected: admission and discharge dates, age, race, ethnicity, HIV serostatus, whether or not the HIV diagnosis was concurrent with PJP diagnosis, HIV risk factor, CD4 count (cells $/ \mathrm{mm}^{3}$ ), CD4 \%, HIV RNA (copies/mL), HIV risk factors, whether or not the patient was on HAART prior to admission, alveolar-arterial (AA) gradient $(\mathrm{mmHg}$ ), arterial $\mathrm{PaO} 2$ in $\mathrm{mmHg}$, oxygen saturation (SpO2) in mmHg, lactate dehydrogenase (LDH) on admission (units/L), whether or not the patient was admitted to the intensive care unit (ICU), ICU LOS, duration of mechanical ventilation, hospital LOS, serum creatinine (mg/dL), total bilirubin $(\mathrm{mg} / \mathrm{dL})$, albumin $(\mathrm{gm} / \mathrm{dL})$, medications used to treat PJP including dosage and duration, corticosteroids used to treat PJP including dosage and duration, and whether or not the patient (a) was initiated on HAART in the hospital, (b) experienced immune reconstitution with inflammatory syndrome (IRIS), (c) expired during hospitalization, and/or (d) experienced treatment failure.

Outpatient HIV clinic records were reviewed to determine duration of and adherence to PJP and corticosteroid treatment. HAART was defined as at least 3 antiretroviral medications from at least 2 drug classes to which the patient's HIV was susceptible by genotypic analysis. A treatment-limiting adverse event was defined as a medication side effect that required medication discontinuation as documented by a physician in the electronic medical record. Severity of disease for each patient was calculated using the Pneumonia Severity Index (PSI). ${ }^{8}$

\section{Outcomes}

Current HIV opportunistic treatment guidelines define PJP treatment failure as worsening of respiratory function or lack of improvement after at least 4-8 days of PJP treatment. ${ }^{5}$ The guidelines also state that worsening of respiratory function should be determined by worsening arterial blood gases and that clinicians should wait at least 4-8 days before changing PJP treatment due to lack of improvement. In the current study, treatment failure was defined by either worsening clinical status (WCS) after at least 5 days of therapy and/or mortality due to PJP. WCS after at least 5 days of therapy was defined as the occurrence of 1 or more of the following: (a) worsening dyspnea based on an infectious diseases physician's assessment, (b) persistently elevated LDH (>250 units/L), (c) worsening in $\mathrm{PaO} 2(<70 \mathrm{mmHg})$ or AA gradient $(>35 \mathrm{mmHg})$ after initial improvement, or (d) change in initial PJP treatment based on a judgment of clinical worsening or inappropriate PJP treatment by an infectious diseases physician as documented in the electronic medical record. Five days was selected as the time for WCS based on PJP treatment guidelines and discussions with board certified infectious diseases physicians at UNMH.

\section{Statistical Analysis}

Patient characteristics and treatment outcomes were summarized using descriptive statistics. Comparisons between PRE and POST were performed using 2 sample t-tests for continuous variables and chi-square or Fisher's exact tests for categorical 


\section{FIGURE 1 CONSORT Flow Diagram} of Study Patients

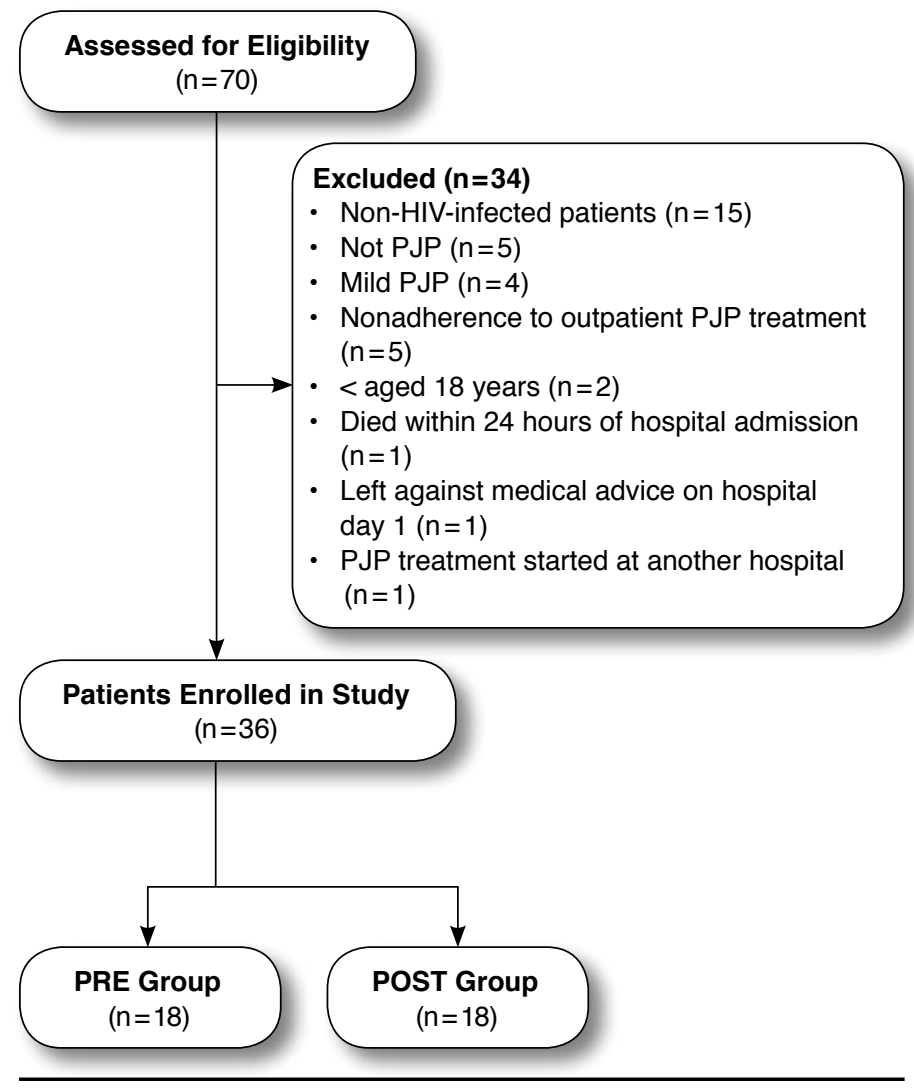

CONSORT $=$ Consolidated Standards of Reporting Trials; HIV = human immunodeficiency virus; $P J P=$ Pneumocystis jirovecii pneumonia; $P O S T=$ postshortage; $P R E=$ preshortage.

variables. Wilcoxon rank sum tests were used for nonparametric data. All tests were two-tailed, and a $P$ value of $\leq 0.05$ was considered statistically significant. All analyses were performed with JMP software, version 10 (SAS Institute, Cary, NC).

\section{Results}

\section{Study Participants}

Medical records for 70 patients included the ICD-9-CM code 136.3 for PJP at UNMH during the study period of which 36 met inclusion criteria (Figure 1). Baseline patient characteristics are shown in Table 1. At time of PJP presentation, 10 out of the 18 patients in each group (55.6\%) had arterial blood gas data, while the other patients had only SpO2 data. Five patients in PRE (27.8\%) and 6 patients in POST (33.3\%) had $\mathrm{SpO} 2$ values on room air. Three patients in PRE (16.7\%) and 2 patients in POST (11.1\%) had SpO2 values above $89 \mathrm{mmHg}$ (range $89 \mathrm{mmHg}-96 \mathrm{mmHg}$ ) while on supplemental oxygen (range 2-15 L/minute). Ten patients in PRE (55.6\%) and 12 patients in POST (66.7\%) had a confirmed PJP diagnosis with a Gomori Methenamine Silver or Calcofluor White stain All other patients in each group had a presumed diagnosis of PJP. A board certified infectious diseases physician was involved in the diagnosis and management of all patients in the study. Many baseline patient characteristics were similar between groups including age, PSI, AA gradient, $\mathrm{PaO} 2$, need for mechanical ventilation, and CD4 count. However, patients in PRE had a significantly higher median (interquartile range [IQR]) LDH level than those in POST $(971$ [803-1,219] and 464 [269-884], respectively, $P=0.038$ ). Patients in PRE were less likely to be on HAART or PJP chemoprophylaxis at the time of PJP diagnosis than patients in POST $(0 \%$ and $16.7 \%$, respectively for HAART, $P=0.035 ; 5.6 \%$ and $22.2 \%$, respectively, for PJP chemoprophylaxis, $P=0.136$ ). Patients in PRE were also less likely to be initiated on HAART during PJP treatment than patients in POST (11.1\% and $38.9 \%$, respectively, $P=0.049)$. No patients who received HAART experienced IRIS.

\section{Outcomes}

Treatment outcomes are shown in Table 2. Three patients in PRE (19\%) and 6 patients in POST (33.3\%) experienced treatment failure $(P=0.248)$. Three patients in each group expired (16.7\%, respectively, $P>0.999)$. No patients in PRE and 3 patients $(16.7 \%)$ in POST experienced WCS but did not expire $(P=0.035)$. All but 1 of the treatment failures in POST $(83.3 \%)$ occurred between June 2010 and November 2010, the first 6 months of the IV TMP/SMX shortage (Figure 2). The only other treatment failure in POST occurred in October 2011 and that patient expired. The 3 patients in PRE who experienced treatment failure were all treated with IV TMP/SMX. All 3 of these patients expired. Median LOS was 11 days (IQR 7-17) in PRE and 14 days (IQR 5-22) in POST $(P=0.800)$. The 3 patients in POST who experienced WCS had a longer median LOS than those patients who did not experience WCS, 18 days (IQR $12-28)$ and 11 days (IQR 5-18), respectively $(P=0.228)$.

\section{PJP Treatment}

PJP treatments used in each group are shown in Table 3 with a detailed list of PJP treatments used in POST shown in Table 4. Sixteen patients in PRE (88.3\%) and 13 patients in POST $(72.2 \%)$ received TMP/SMX therapy $(P=0.200)$. In PRE, 7 patients (38.9\%) were started on oral PJP treatment compared with $13(72.2 \%)$ in POST $(P=0.042)$. In PRE, 2 patients $(11.1 \%)$ received oral TMP/SMX therapy during April 2010, the month before the IV TMP/SMX shortage occurred. Additionally, of the patients started on oral therapy, 1 patient in PRE (14.3\%) and 4 in POST $(30.8 \%)$ were transitioned to IV therapy $(P=0.402)$. The median TMP/SMX dose was slightly higher in POST compared with PRE, but the difference was not statistically significant (15.6 mg/kg [13-20.7]) and $15 \mathrm{mg} / \mathrm{kg}$ [14.1-15.8], respectively, $P=0.546)$. In POST, 16 patients received oral TMP/SMX, 13 as initial therapy and 3 after initial therapy, and all but 3 of these patients (81.3\%) received a dose of at least $15 \mathrm{mg} / \mathrm{kg}$ of 


\section{TABLE 1 Patient Demographic and Clinical Characteristics ${ }^{a}$}

\begin{tabular}{|c|c|c|c|c|c|}
\hline \multirow[b]{2}{*}{ Variable } & \multicolumn{5}{|c|}{ Treatment Group } \\
\hline & \multicolumn{2}{|c|}{$\operatorname{PRE}(n=18)$} & \multicolumn{2}{|c|}{$\operatorname{POST}(n=18)$} & $P$ Value \\
\hline \multicolumn{6}{|l|}{ Demographics } \\
\hline Age (years), mean [SD] & 42 & [9] & 38 & [12] & $0.250^{\mathrm{b}}$ \\
\hline Male sex, \% (n) & 100.0 & (18) & 94.4 & (17) & 0.234 \\
\hline Race, \% (n) & & & & & 0.377 \\
\hline Caucasian & 33.3 & (6) & 44.4 & (8) & \\
\hline Hispanic & 16.7 & (3) & 27.8 & $(5)$ & \\
\hline Otherc $^{c}$ & 50.0 & (9) & 27.8 & (5) & \\
\hline HIV risk factor & & & & & 0.296 \\
\hline Sexual contact & 55.6 & (10) & 72.2 & (13) & \\
\hline IDU or unknown & 44.4 & $(8)$ & 27.8 & (5) & \\
\hline CD4 count (cells/mm³), median (IQR) & 10 & $(6-47)$ & 38 & $(10-71)$ & $0.268^{\mathrm{d}}$ \\
\hline CD4 \%, median (IQR) & 3 & $(1-7)$ & 4 & $(2-8)$ & $0.773^{\mathrm{d}}$ \\
\hline HIV RNA (copies/mL), median (IQR) & $435,000(152$ & $00-765,000)$ & $225,000(44,7$ & $-1,575,000)$ & $0.496^{\mathrm{d}}$ \\
\hline New HIV diagnosis, \% (n) & 50.0 & (9) & 55.6 & (10) & 0.734 \\
\hline PJP prophylaxis prior to diagnosis & 5.6 & (1) & 22.2 & (4) & 0.136 \\
\hline HAART prior to diagnosis & 0.0 & $(0)$ & 16.7 & (3) & 0.035 \\
\hline PJP diagnosis, \% (n) & & & & & 0.494 \\
\hline Calcofluor White or GMS & 55.6 & (10) & 66.7 & (12) & \\
\hline Clinician judgment & 44.4 & (8) & 33.3 & (6) & \\
\hline PJP sample, \% (n) & & & & & 0.717 \\
\hline BAL or tissue biopsy & 66.7 & $(12)$ & 72.2 & (13) & \\
\hline No sample obtained & 33.3 & (6) & 27.8 & (5) & \\
\hline \multicolumn{6}{|l|}{ Disease severity } \\
\hline Alveolar/arterial gradient $(\mathrm{mmHg})$, mean $[\mathrm{SD}]^{\mathrm{e}}$ & 61 & [17] & 56 & [10] & $0.456^{b}$ \\
\hline $\mathrm{Pa02}(\mathrm{mmHg})$, mean $[\mathrm{SD}]^{\mathrm{e}}$ & 56 & [17] & 51 & [15] & $0.525^{b}$ \\
\hline Room air SpO2 $(\mathrm{mmHg})$, mean $[\mathrm{SD}]^{\mathrm{f}}$ & 80 & {$[7]$} & 78 & [7] & \\
\hline Pneumonia Severity Index, median (IQR) & 71 & $(55-96)$ & 56 & $(38-117)$ & $0.342^{\mathrm{d}}$ \\
\hline Serum creatinine (mg/dL), median (IQR) & 0.95 & $(0.79-1.22)$ & 0.89 & $(0.74-1.05)$ & $0.393^{d}$ \\
\hline LDH (units/L), median (IQR) & 971 & $(803-1,219)$ & 464 & $(269-884)$ & $0.006^{\mathrm{d}}$ \\
\hline Albumin (gm/dL), mean [SD] & 2.9 & {$[0.6]$} & 2.9 & {$[0.6]$} & $0.827^{\mathrm{b}}$ \\
\hline Total bilirubin (mg/dL), median (IQR) & 0.5 & $(0.5-0.8)$ & 0.4 & $(0.3-0.9)$ & $0.135^{\mathrm{d}}$ \\
\hline Intensive care unit admission, \% (n) & 33.3 & (6) & 50.0 & (9) & 0.309 \\
\hline Mechanical ventilation, \% (n) & 33.3 & (6) & 27.8 & (5) & 0.717 \\
\hline
\end{tabular}

${ }^{a}$ Chi-squared test was utilized unless otherwise noted. All tests were two-tailed, and a P value of $\leq 0.05$ was considered statistically significant.

bTwo sample t-test.

${ }^{c}$ Comprised Asian/Pacific Islander, African American, Native American/Alaskan Native, 2 or more races, and unknown/declined to answer.

dWilcoxon rank sum test.

'Ten patients in each group had arterial blood gas data at the time of PJP presentation.

fFive patients in PRE and 6 patients in POST had SpO2 data on room air at time of PJP presentation. Three patients in PRE and 2 patients in POST had SpO2 data on supplemental oxygen at the time of PJP presentation.

$B A L=$ bronchoaveolar lavage; gm/dL= gram per deciliter; GMS= Gormori Methenamine Silver stain; HAART=highly active antiretroviral therapy; HIV=human immuno-

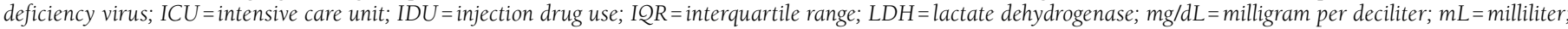
$\mathrm{mm}^{3}=$ cubic millimeter; $\mathrm{mmHg}=$ millimeter of mercury; $\mathrm{PaO2}=$ arterial partial pressure of oxygen; $P J P=$ Pneumocystis jirovecii pneumonia; $P O S T=$ postshortage group; $P R E=$ preshortage group; $S D=$ standard deviation; Sp02 = oxygen saturation .

TMP per day. The minimum duration of PJP treatment among those patients who did not expire was 15 days in both groups. Median duration of corticosteroid treatment was similar between PRE and POST, as well (21 [14-21] and 21 [21-21], respectively, $P=0.546)$.
Both IV pentamidine and IV clindamycin with oral primaquine were used in POST. IV pentamidine was not used in PRE, but this medication was administered to 6 patients in POST (33.3\%) - twice as empiric therapy and 4 times as second-line therapy after a course of oral therapy (oral TMP/SMX 


\section{TABLE 2 Pneumocystis jirovecii Pneumonia Treatment Outcomes ${ }^{a}$}

\begin{tabular}{|c|c|c|c|c|c|}
\hline \multirow{3}{*}{$\begin{array}{l}\text { Variable } \\
\text { Treatment failure, \% (n) }\end{array}$} & \multicolumn{5}{|c|}{ Treatment Group } \\
\hline & \multicolumn{2}{|c|}{$\operatorname{PRE}(n=18)$} & \multicolumn{2}{|c|}{ POST $(n=18)$} & \multirow{2}{*}{$\begin{array}{c}P \text { Value } \\
0.248\end{array}$} \\
\hline & 16.7 & (3) & 33.3 & (6) & \\
\hline Worsening clinical status, \% (n) & 0.0 & (0) & 16.7 & (3) & 0.035 \\
\hline Death due to PJP, \% (n) & 16.7 & (3) & 16.7 & (3) & $>0.999$ \\
\hline LOS (days), median (IQR) & 11.0 & $(7-17)$ & 14.0 & $(5-22)$ & $0.800^{\mathrm{b}}$ \\
\hline Duration of mechanical ventilation (days), mean [SD] & 9.0 & [5] & 10.0 & [6] & $0.927^{c}$ \\
\hline
\end{tabular}

${ }^{a}$ Chi-squared test was utilized unless otherwise noted. All tests were two-tailed, and a P value of $\leq 0.05$ was considered statistically significant.

${ }^{b}$ Wilcoxon rank sum test.

'Two sample t-test.

$I Q R=$ interquartile range; $L O S=$ length of stay; $P J P=$ Pneumocystis jirovecii pneumonia; $P O S T=$ postshortage group; $P R E=$ preshortage group; $S D=$ standard deviation

\section{FIGURE 2 PJP Treatment Failures over Time}

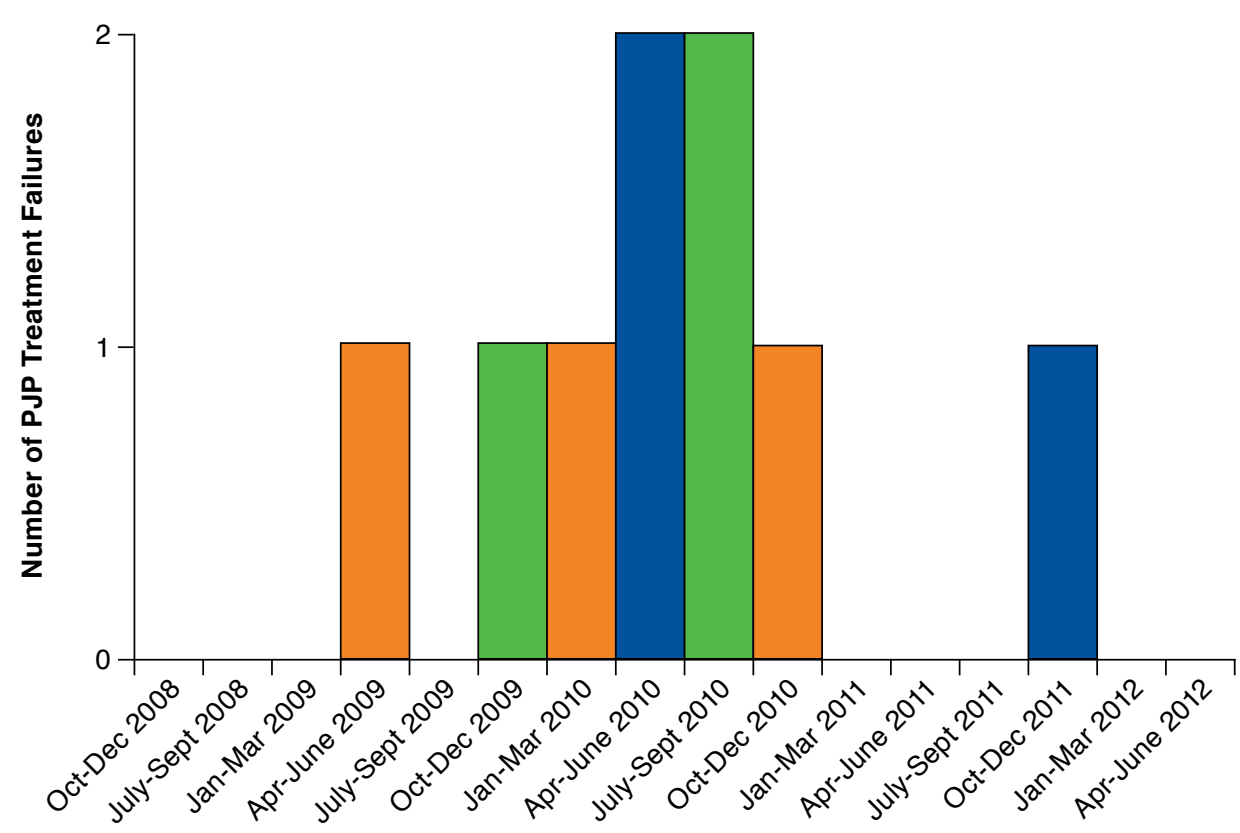

aThe intravenous trimethoprim-sulfamethoxazole shortage began June 1, 2010. PJP=Pneumocystis jirovecii pneumonia.

for 3 patients and IV clindamycin with oral primaquine for 1 patient). IV pentamidine had to be stopped due to treatmentlimiting adverse events in 3 patients. Clindamycin (either IV or oral) with oral primaquine was administered 6 times to 5 patients in POST. This combination was administered 3 times as empiric therapy (IV) and 3 times as second-line therapy after a patient experienced WCS with oral TMP/SMX (1 patient), WCS with IV pentamidine (1 patient), and successful with IV pentamidine (1 patient). One patient received IV clindamycin with oral primaquine twice. This patient was started on IV clindamycin with oral primaquine, transitioned to IV pentami- dine, experienced WCS, and was transitioned to IV clindamycin with oral primaquine. No patients experienced treatmentlimiting side effects with clindamycin and primaquine. One patient in POST received IV clindamycin with oral atovaquone after experiencing side effects due to IV pentamidine. This patient had a documented intolerance to TMP/SMX, but after 8 days of IV clindamycin with oral atovaquone, this patient was changed to oral TMP/SMX. All doses of clindamycin, primaquine, pentamidine, and atovaquone were consistent with current HIV opportunistic infection treatment guidelines for PJP. ${ }^{5}$ 


\begin{tabular}{|c|c|c|c|c|c|}
\hline \multirow[b]{2}{*}{ Variable } & \multicolumn{5}{|c|}{ Treatment Group } \\
\hline & \multicolumn{2}{|c|}{ PRE $(n=18)$} & \multicolumn{2}{|c|}{$\operatorname{POST}(n=18)$} & \multirow{2}{*}{$\frac{P \text { Value }}{0.200}$} \\
\hline & & & & & \\
\hline TMP/SMX' & 88.8 & (16) & 72.2 & (13) & \\
\hline Otherc & 11.2 & (2) & 27.8 & $(5)$ & \\
\hline Initial TMP/SMX dose (mg/kg/day), median [IQR] & 15.0 & (14.1-15.8) & 15.6 & $(13-20.7)$ & $0.546^{\mathrm{d}}$ \\
\hline Oral therapy used initially, \% (n) & 38.9 & $(7)$ & 72.2 & (13) & 0.042 \\
\hline Change from initial oral therapy to IV therapy, \% (n) & 5.6 & (1) & 22.2 & (4) & 0.136 \\
\hline Duration anti-PJP therapy (days), median [IQR] & 21.0 & $(20-21)$ & 21.0 & $(21-21)$ & $0.640^{\mathrm{d}}$ \\
\hline Duration corticosteroid therapy (days), median [IQR] & 21.0 & $(14-21)$ & 21.0 & $(18-21)$ & 0.981 \\
\hline $\begin{array}{l}{ }^{a} \text { Chi-squared test was used for all statistical analyses. All t } \\
{ }^{b} \text { Includes oral and IV formulations. } \\
{ }^{c} \text { Comprised IV pentamidine and IV clindamycin with oral } \\
{ }^{d} \text { Wilcoxon rank sum test. } \\
\text { IQR = interquartile range; IV=intravenous; } \mathrm{kg}=\text { kilogram; } n \\
\text { TMP/SMX = trimethoprim/sulfamethoxazole. }\end{array}$ & Pneume & cystis jiroveci & POST $=$ & ostshortage & eshortage \& \\
\hline
\end{tabular}

\section{Discussion}

This is the first study attempting to address the impact of the IV TMP/SMX shortage on treatment outcomes among HIVinfected patients with moderate-to-severe PJP. The results of the study show that the IV TMP/SMX shortage may have led to an immediate but temporary negative impact on PJP treatment outcomes. No patients before the shortage or after the first 6 months of the shortage experienced WCS. The mortality rate in both groups was $16.7 \%$, which is similar to a recently published observational study examining PJP treatment outcomes in the era of HAART. ${ }^{9}$ Patients with PJP in a study of PJP treatment outcomes by Fei et al. (2009) had a median LOS of 8 days. ${ }^{10}$ This number is comparable with patients in PRE, but patients in POST had a longer median LOS, especially patients who experienced WCS. When examined together, these data suggest that the lack of IV TMP/SMX may have led to a temporary increase in PJP-related morbidity and LOS but not necessarily an increase in PJP-related mortality. The patients who experienced WCS in POST were treated with either IV pentamidine or IV clindamycin with oral primaquine after receiving at least 5 days of therapy with oral TMP/SMX or IV pentamidine. These patients' clinical course may have been different had IV TMP/SMX been available. Patients in both PRE and POST had similar baseline levels of immunosuppression, respiratory compromise, and need for mechanical ventilation, making the results of the current study unlikely to have resulted for intergroup variability on these variables. However, there were some differences in baseline characteristics that may indicate that patients in POST were poised to experience better PJP treatment outcomes than patients in PRE.

Patients in PRE had a higher LDH than patients in POST, and elevated LDH has been shown to be prognostic of poor PJP treatment outcomes. ${ }^{11-13}$ Other studies have found that elevated LDH does not correlate with a worse PJP treatment outcome..$^{14,15}$ Much of these LDH data are from the pre-HAART era and may not be generalizable to patients in this study. Patients in POST were more likely than patients in PRE to be on HAART or initiated on HAART during their hospitalizations. HAART has been shown to improve PJP treatment outcomes in HIV-infected patients, and current HIV opportunistic infection treatment guidelines recommend initiating HAART within 2 weeks of initiating PJP treatment. ${ }^{3,16,17}$ The use of PJP chemoprophylaxis was more common in POST, as well. In another recent study of HIV-infected patients with PJP by Lim et al. (2012), patients who were not on PJP prophylaxis were 10.8 times more likely to die from PJP than patients on PJP prophylaxis $(P<0.001) .{ }^{18}$ While a mortality difference was not observed between groups in the current study, these patientlevel differences between groups may indicate that patients in POST were more likely to experience better treatment outcomes than patients in PRE. However, patients in POST experienced worse treatment outcomes, and this was likely due to the antimicrobials available to treat PJP in each group.

Current guidelines recommend the use of TMP/SMX as first-line therapy for PJP, but only $72 \%$ of patients in POST were treated with TMP/SMX despite excluding patients with a TMP/SMX allergy. All patients who received TMP/SMX in POST received oral TMP/SMX, and more than 20\% of those patients had to be transitioned to alternative IV therapies. IV treatment is recommended for hospitalized patients with moderate-to-severe PJP until clinical improvement occurs. ${ }^{5}$ IV pentamidine is an alternative to TMP/SMX, but many patients develop treatment-limiting adverse events with IV pentamidine. ${ }^{19}$ Treatment-limiting adverse events were observed among patients treated with IV pentamidine in the current study, as well. IV clindamycin with primaquine may be an effective salvage therapy, and this regimen was an effective second-line therapy among patients in POST. ${ }^{20,21}$ Clindamycin with oral 
Impact of an Intravenous Trimethoprim/Sulfamethoxazole Shortage on Treatment

Outcomes Among HIV-Infected Patients with Pneumocystis jirovecii Pneumonia

\section{TABLE 4 Treatment and Outcomes in POST}

\begin{tabular}{|c|c|c|c|c|c|}
\hline Patient & First-Line Treatment & Second-Line Treatment & Third-Line Treatment & TMP Dose ${ }^{a}$ & Treatment Outcome \\
\hline 1 & PO TMP/SMX, 13 days & IV Pentam, 8 days & & 22.2 & Mortality \\
\hline 2 & IV C $+\mathrm{P}, 2$ days & & & N/A & Mortality \\
\hline 3 & PO TMP/SMX, 21 days & & & 13.7 & Resolution \\
\hline 4 & PO TMP/SMX, 13 days & IV Pentam, 8 days & & 22.5 & Resolution \\
\hline 5 & PO TMP/SMX, 10 days & IV Pentam, 4 days & $\mathrm{PO} \mathrm{C}+\mathrm{P}, 16$ days & 15.1 & WCS (TMP) \\
\hline 6 & IV C $+\mathrm{P}, 2$ days & IV Pentam, 7 days & IV C+P, 14 days & N/A & WCS (Pentam) \\
\hline 7 & PO TMP/SMX, 18 days & & & 16.3 & Resolution \\
\hline 8 & PO TMP/SMX, 5 days & IV C+P, 4 days & PO TMP/SMX, 9 days & 17.5 & WCS (TMP) \\
\hline 9 & PO TMP/SMX, 21 days & & & 17.1 & Resolution \\
\hline 10 & PO TMP/SMX, 21 days & & & 14.1 & Resolution \\
\hline 11 & IV Pentam, 2 days & PO TMP/SMX, 20 days & & 16.1 & Resolution \\
\hline 12 & PO TMP/SMX, 21 days & & & 15.0 & Resolution \\
\hline 13 & IV Pentam, 4 days & IV C+Atova, 8 days & PO TMP/SMX, 19 days & 12.8 & Mortality \\
\hline 14 & IV C+P, 2 days & PO TMP/SMX, 19 days & & 22.3 & Resolution \\
\hline 15 & PO TMP/SMX, 18 days & & & 15.7 & Resolution \\
\hline 16 & PO TMP/SMX, 5 days & Atova, 16 days & & 16.6 & Resolution \\
\hline 17 & PO TMP/SMX, 20 days & & & 15.0 & Resolution \\
\hline 18 & PO TMP/SMX, 21 days & & & 21.8 & Resolution \\
\hline
\end{tabular}

aTrimethoprim doses are expressed as $\mathrm{mg} / \mathrm{kg} / \mathrm{day}$. The recommended trimethoprim dose for moderate-to-severe Pnuemocystis jirovecii pneumonia in HIV-infected patients is $15-20 \mathrm{mg} / \mathrm{kg} / \mathrm{day} .^{5}$

Atova $=$ oral atovaquone; $C+$ Atova $=$ clindamycin with oral atovaquone; $C+P=$ clindamycin with oral primaquine; HIV=human immuneodeficiency virus;

$I V=$ intravenous; $\mathrm{mg} / \mathrm{kg} /$ day = milligram per kilogram per day; $\mathrm{N} / \mathrm{A}=$ not applicable; Pentam = pentamidine; $P O=$ oral; $P O S T=$ postshortage group; $T M P=$ trimethoprim;

TMP/SMX = trimethoprim/sulfamethoxazole; WCS $(P)=$ worsening clinical status while on IV pentamidine; WCS $(T M P)=$ worsening clinical status while on oral TMP/SMX.

primaquine was also better tolerated than IV pentamidine in the current study. These alternative PJP treatments were somewhat effective in POST but were a poor substitute for IV TMP/ SMX.

Our results are consistent with a recent survey of infectious diseases physicians in which 55\% of respondents reported adverse patient outcomes due to antimicrobial shortages, including long-term morbidity from inadequate treatment and increased LOS (28\% and 26\%, respectively). ${ }^{22}$ The number one antimicrobial reported as being unavailable by infectious diseases physicians in the survey was IV TMP/SMX (65\% of respondents). ${ }^{22}$ Drug shortages have become common among many classes of medication, but the number of antimicrobials impacted by drug shortages poses a serious problem for the provision of anti-infective therapy in the United States..$^{23,24}$ A recent survey of pharmacy directors by McLaughlin et al. (2013) sought to measure the impact of drug shortages on patient outcomes, clinical pharmacy operations, patient complaints, and institutional cost. ${ }^{25}$ The authors found that $85.3 \%$ of respondents reported the use of alternative medications; $70.8 \%$ reported delays in medication therapy; and almost 50\% reported a need for increased patient monitoring. The use of alternative therapies and the need for increased monitoring were observed during the study period. However, in our study, delays in care were not common as patients were immediately started on alternative PJP treatments in the absence of IV TMP/ SMX. When examining our results, it is important to consider that June 1, 2010, is a conservative estimate of when the IV
TMP/SMX shortage began. This date was selected for this study based on conversations with pharmacy leadership at UNMH, but we were unable to ascertain whether or not the pharmacy department was rationing the use of IV TMP/SMX before June 1, 2010. For example, of the few patients who received oral TMP/SXM in PRE, 2 of them received oral TMP/SMX in April and May 2010. May 2010 has been cited by some as the beginning of the IV TMP/SMX shortage in the United States. ${ }^{23}$

While the lack of IV TMP/SMX may have led to poor treatment outcomes immediately following the shortage, this trend leveled off after 6 months. This was likely due to physician and pharmacist awareness of the drug shortage and subsequent interventions to improve patient outcomes. For example, UNMH infectious diseases-trained pharmacists led an educational campaign to ensure that oral doses of TMP/SMX met the recommended $15-20 \mathrm{mg} / \mathrm{kg} / \mathrm{day}$ of TMP and to inform clinicians of alternatives to IV TMP/SMX. ${ }^{5}$ This educational campaign included didactic presentations by infectious diseases-trained pharmacists to faculty and residents within the departments of Infectious Diseases, Critical Care, Trauma Medical, Neurosurgical, and Trauma/Surgical Critical Care. Infectious diseases-trained pharmacists also gave didactic presentations to pharmacists, pharmacy residents, and pharmacy students working at UNMH. The UNMH Antimicrobial Stewardship Pharmacist also reviewed all oral TMP/SMX dosing during POST and made recommendations that prescribers increase doses to the recommended $15-20 \mathrm{mg} / \mathrm{kg}$ of TMP per day if the dose was too low. Finally, all HIV-infected patients at 
UNMH are seen by the Infectious Diseases consult team, which rounds with an infectious diseases-trained pharmacist. These pharmacists would recommend appropriate doses of oral TMP/ SMX while on rounds with the consult team. The median TMP/ SMX dose was slightly higher in POST, which is likely attributable to these pharmacist-led educational initiatives. Using appropriate doses of oral TMP/SMX was a priority at UNMH because of the educational interventions led by the infectious diseases-trained pharmacists. As the shortage continued, these pharmacists also educated infectious diseases physicians on the appropriate dosing of alternative anti-PJP treatments and how to manage side effects associated with these agents. Providers at UNMH then became more familiar with IV pentamidine and managing its side effects. Additionally, patients in POST may have been more likely to experience better treatment outcomes because of the use of HAART and PJP prophylaxis. These educational efforts coupled with HAART and PJP prophylaxis may have lessened the impact of the IV TMP/SMX shortage on treatment outcomes at our facility over time.

\section{Limitations}

While we attempted to cast light on this drug shortage, this was a retrospective study performed at a single academic medical center, and our results may not be generalizable to other institutions. Considerable rigor was put into the design of the current study to ensure all assessments and outcomes were valid and well supported. The small sample size is a limitation, as well, and makes the statistical analysis and subsequent interpretation more challenging than would be with a larger sample size. With the number of patients and the observed failure proportions in the 2 groups, we had less than 20\% power to detect a difference in treatment failure between the 2 groups at alpha $=0.05 ; 106$ patients per group would provide $80 \%$ power to detect a difference in the proportion of treatment failure using alpha $=0.05$. However, PJP in HIV-infected patients is becoming less common as the threshold for initiating HAART continues to decrease. ${ }^{4,26}$ We believe that the current sample size is within expectation for a large academic medical center over an almost 4-year period and is comparable with a recent observational study of PJP. ${ }^{9}$ Moreover, the objective of this study was to evaluate the impact of the shortage on PJP treatment outcomes among HIV-infected patients, and we believe this objective was achieved.

\section{Conclusions}

A national shortage of IV TMP/SMX may have led to increased patient morbidity and LOS among HIV-infected patients with moderate-to-severe PJP, particularly in the 6 months immediately following the drug shortage at our institution. Pharmacist-led educational interventions along with more frequent use of PJP chemoprophylaxis and antiretroviral therapy may have helped mitigate the impact of this drug shortage on
HV-infected patient treatment outcomes when IV TMP/SMX was not available. While the incidence of PJP among HIVinfected patients is decreasing, PJP still affects HIV-infected patients, as well as patients with other immunosuppressive conditions and patients on immunosuppressive medications. ${ }^{27,28}$ Patients without HIV who develop PJP experience worse treatment outcomes than HIV-infected patients. ${ }^{26,27}$ IV TMP/SMX is also used to treat infections caused by other pathogens, such as Stenotrophomonas maltophilia and Nocardia species. ${ }^{29}$ Production of IV TMP/SMX has resumed, but there must be a concerted effort to avoid shortages of IV TMP/SMX in the future. Future studies should evaluate the impact of this shortage using larger databases and should include nonHIV-infected patients. Future studies should also evaluate how pharmacists, physicians, and health systems respond to drug shortages that affect HIV-infected patients.

\section{Authors}

THOMAS J. DILWORTH, PharmD, BCPS, AAHIVP, is Clinical Pharmacy Supervisor, Wheaton Franciscan Healthcare, St. Francis Hospital, Milwaukee, Wisconsin; OMAR M. IBRAHIM, PhD, is Assistant Professor of Pharmacotherapy, Department of Pharmacy, Faculty of Pharmacy, Al-Zaytoonah University of Jordan, Amman, Jordan; and RENÉE-CLAUDE MERCIER, PharmD, BCPS, PhC, is Associate Professor of Pharmacy and Medicine, Department of Pharmacy Practice and Administrative Sciences, University of New Mexico Health Sciences Center College of Pharmacy, Albuquerque, New Mexico.

AUTHOR CORRESPONDENCE: Renée-Claude Mercier, PharmD, BCPS, PhC, University of New Mexico College of Pharmacy, 2502 Marble N.E., MSC 09 5360, Albuquerque, NM 87131-0001.

Tel.: 505.272.9553; Fax: 505.272.6749; E-mail: rmercier@unm.edu.

\section{DISCLOSURES}

Dilworth and Ibrahim have nothing to disclose. Mercier has provided expert testimony for the U.S. Department of Justice. This work was supported by the University of New Mexico College of Pharmacy. No direct funding was received for this study. No funding bodies had any role in the study design, data collection, analysis, decision to publish, or preparation of the manuscript. This research was presented in part at the European Congress of Clinical Microbiology and Infectious Diseases (Abstract number 3600), Berlin, Germany, April 27, 2013.

At the time of manuscript development, Dilworth was an Infectious Diseases Pharmacotherapy Research Fellow and Ibrahim was an Infectious Diseases Postdoctoral Researcher at the University of New Mexico College of Pharmacy.

Dilworth was responsible for concept and design, along with Ibrahim and Mercier. Dilworth collected the data, which were interpreted equally by all authors. The manuscript was written primarily by Dilworth, assisted by Ibrahim and Mercier. All authors worked equally on manuscript revision. 


\section{REFERENCES}

1. Kovacs JA, Masur H. Evolving health effects of Pneumocystis: one hundred years of progress in diagnosis and treatment. JAMA. 2009;301(24):2578-85.

2. Thomas CF Jr, Limper AH. Pneumocystis pneumonia. N Engl J Med. 2004;350(24):2487-98. Available at: http://www.nejm.org/doi/pdf/10.1056/ NEJMra032588. Accessed September 23, 2014

3. Phair J, Muñoz A, Detels R, Kaslow R, Rinaldo C, Saah A. The risk of Pneumocystis carinii pneumonia among men infected with human immunodeficiency virus type 1. Multicenter AIDS Cohort Study Group. N Engl J Med. 1990;322(3):161-65. Available at: http://www.nejm.org/doi/pdf/10.1056/ NEJM199001183220304. Accessed September 23, 2014

4. Buchacz K, Baker RK, Palella FJ Jr, et al. AIDS-defining opportunistic illnesses in U.S. patients, 1994-2007: a cohort study. AIDS. 2010;24(10):1549-59.

5. Panel on Opportunistic Infections in HIV-Infected Adults and Adolescents. Guidelines for the prevention and treatment of opportunistic infections in HIV-infected adults and adolescents: recommendations from the Centers for Disease Control and Prevention, the National Institutes of Health, and the HIV Medicine Association of the Infectious Diseases Society of America. Available at: http://aidsinfo.nih.gov/contentfiles/lvguidelines/ adult_oi.pdf. Accessed September 23, 2014. [pp. B1-B18]

6. American Society of Health-System Pharmacists. Drug shortages. Sulfamethoxazole/trimethoprim injection. Updated April 16, 2014. Available at: http://www.ashp.org/menu/DrugShortages/CurrentShortages/Bulletin. aspx?id=613. Accessed October 1, 2014.

7. von Elm E, Altman DG, Egger M, et al. The strengthening the reporting of observational studies in epidemiology (STROBE) statement: guidelines for reporting observational studies. Prev Med. 2007;45(4):247-51.

8. Fine MJ, Singer DE, Hanusa BH, Lave JR, Kapoor WN. Validation of a pneumonia prognostic index using the MedisGroups Comparative Hospital Database. Am J Med. 1993;94(2):153-19.

9. Libre JM, Revollo B, Vanegas S, et al. Pneumocystis jirovecii pneumonia in HIV-l-infected patients in the late-HAART era in developed countries. Scan J Infect Dis. 2013;45(8):635-44.

10. Fei MW, Kim EJ, Sant CA, et al. Predicting mortality from HIVassociated Pneumocystis pneumonia at illness presentation: an observational cohort study. Thorax. 2009;64(12):1070-76. Available at: http://thorax.bmj. com/content/64/12/1070.full.pdf+html. Accessed September 23, 2014.

11. Speich R, Weber R, Kronauer CM, Opravil M, Lüthy R, Russi EW. Prognostic score for Pneumocystis carinii pneumonia. Respiration. 1990;57(4):259-63

12. Benson CA, Spear J, Hines D, Pottage JC Jr, Kessler HA, Trenholme GM. Combined APACHE II score and serum lactate dehydrogenase as predictors of in-hospital mortality caused by first episode Pneumocystis carinii pneumonia in patients with acquired immunodeficiency syndrome. Am Rev Respir Dis. 1991;144(2):319-23.

13. Speich R, Opravil M, Weber R, Hess T, Luethy R, Russi EW. Prospective evaluation of a prognostic score for Pneumocystis carinii pneumonia in HIVinfected patients. Chest. 1992;102(4):1045-48. Available at: http://journal. publications.chestnet.org/data/Journals/CHEST/21656/1045.pdf. Accessed September 23, 2014

14. Vanhems P, Toma E. Evaluation of a prognostic score. Pneumocystis carinii pneumonia in HIV-infected patients. Chest. 1995;107(1):107-12. Available at: http://journal.publications.chestnet.org/data/Journals/CHEST/21706/107. pdf. Accessed September 23, 2014.
15. Bédos JP, Hignette C, Lucet JC, et al. Serum carcinoembryonic antigen: a prognostic marker in HIV-related Pneumocystis carinii pneumonia. Scand J Infect Dis. 1992;24(3):309-15

16. Morris A, Wachter RM, Luce J, Turner J, Huang L. Improved survival with highly active antiretroviral therapy in HIV-infected patients with severe Pneumocystis carinii pneumonia. AIDS. 2003;17(1):73-80.

17. Zolopa A, Andersen J, Powderly W, et al. Early antiretroviral therapy reduces AIDS progression/death in individuals with acute opportunistic infections: a multicenter randomized strategy trial. PLoS One. 2009;4(5):e5575. Available at: http://www.plosone.org/article/fetchObject.act ion?uri=info\%3Adoi\%2F10.1371\%2Fjournal.pone.0005575\&representation= PDF. Accessed September 23, 2014.

18. Lim PL, Zhou J, Ditangco RA, et al. Failure to prescribe pneumocystis prophylaxis is associated with increased mortality, even in the cART era: results from the Treat Asia HIV observational database. J Int AIDS Soc 2012;15(1):1. Available at: http://www.ncbi.nlm.nih.gov/pmc/articles/ PMC3354658/pdf/1758-2652-15-1.pdf. Accessed September 23, 2014

19. Sattler FR, Cowan R, Nielsen DM, Ruskin J. Trimethoprimsulfamethoxazole compared with pentamidine for treatment of Pneumocystis carinii pneumonia in the acquired immunodeficiency syndrome. A prospective, noncrossover study. Ann Intern Med. 1988;109(4):280-87.

20. Smego RA Jr, Nagar S, Maloba B, Popara M. A meta-analysis of salvage therapy for Pneumocystis carinii pneumonia. Arch Intern Med. 2001;161(12):1529-33. Available at: http://archinte.jamanetwork.com/article. aspx?articleid=648429. Accessed September 23, 2014.

21. Benfield T, Atzori C, Miller RF, Helweg-Larsen J. Second-line salvage treatment of AIDS-associated Pneumocystis jirovecii pneumonia: a case series and systematic review. J Acquir Immune Defic Syndr. 2008;48(1):63-67.

22. Gundlapalli AV, Beekmann SE, Graham DR, Polgreen PM. Perspectives and concerns regarding antimicrobial agent shortages among infectious disease specialists. Diagn Microbiol Infect Dis. 2013;75(3):256-59.

23. Griffith MM, Gross AE, Sutton SH, et al. The impact of anti-infective drug shortages on hospitals in the United States: trends and causes. Clin Infect Dis. 2012;54(5):684-91. Available at: http://cid.oxfordjournals.org/content/54/5/684.full.pdf+html. Accessed September 23, 2014.

24. Griffith MM, Patel JA, Sutton SH, et al. Prospective approach to managing antimicrobial drug shortages. Infect Control Hosp Epidemiol. 2012;33(7):745-52.

25. McLaughlin M, Kotis D, Thomson K, et al. Effects on patient care caused by drug shortages: a survey. J Manag Care Pharm. 2013;19(9):78388. Available at: http://www.amcp.org/WorkArea/DownloadAsset. aspx?id=17307.

26. Panel on Antiretroviral Guidelines for Adults and Adolescents Guidelines for the use of antiretroviral agents in HIV-1-infected adults and adolescents. Department of Health and Human Services. Last updated May 1, 2014. Available at: http://aidsinfo.nih.gov/ContentFiles/ AdultandAdolescentGL.pdf. Accessed September 23, 2013

27. Enomoto T, Azuma A, Kohno A, et al. Differences in the clinical characteristics of Pneumocystis jirovecii pneumonia in immunocompromized patients with and without HIV infection. Respirology. 2010;15(1):126-31.

28. Li MC, Lee NY, Lee CC, Lee HC, Chang CM, Ko WC. Pneumocystis jiroveci pneumonia in immunocompromised patients: delayed diagnosis and poor outcomes in non-HIV-infected individuals. J Microbiol Immunol Infect. 2014;47(1):42-47.

29. Gilbert DN, Moellering RC, Eliopoulos GM, Chambers HF, Saag MS. The Sanford Guide to Antimicrobial Therapy 2013. 43rd ed. Sperryville, VA: Antimicrobial Therapy, Inc.; 2013. 